\title{
Parametric correlations of scattering phase shifts and fluctuations of delay times in few-channel chaotic scattering
}

\author{
Yan V. Fyodorovt and Hans-Jürgen Sommers \\ Fachbereich Physik, Universität-Gesamthochschule Essen \\ Essen 45117, Germany
}

(September 15, 2018)

\begin{abstract}
By using the supersymmetry method we derive an explicit expression for the parametric correlation function of densities of eigenphases $\theta_{a}$ of the S-matrix in a chaotic quantum system with broken time-reversal symmetry coupled to continua via $\mathrm{M}$ equivalent open channels; $a=1, . ., M$.We use it to find the distribution of derivatives of these eigenphases over the energy ("phaseshift times") as well as over an arbitrary external parameter.We also find the parametric correlations of Wigner-Smith delay times.
\end{abstract}

PACS numbers: $05.45 .+\mathrm{b}, 24.30 \mathrm{v}$

The most fundamental object characterizing the process of quantum scattering is the unitary S-matrix relating the amplitudes of waves incoming onto the system and the amplitudes of scattered (outgoing) waves.

Recently there was a considerable growth of interest in the phenomenon of quantum chaotic scattering ( see reviews [1], [2]), both theoretically [3, 4, 6, 7], and experimentally 8,9]. Due to the chaotic nature of the underlying scattering dynamics the S-matrix characteristics behave in an irregular way when parameters of either incoming waves (e.g. the energy) or characteristics of the scattering region (e.g. the form and strength of the scattering potential, the strength of the magnetic field through the ballistic microstructure, etc.) are slightly changed. Because of this fact it seems to be most adequate to describe such a behaviour in terms of some statistical measures: distributions and correlation functions.

At present, there are two complementary theoretical tools employed to study open quantum systems whose closed classical counterparts demonstrate chaotic behaviour. These are the semiclassical approach [1] 3] and the stochastic approach [4 6]. The latter one is based on the notion of the universality of the chaotic scattering phenomenon.

It is well known that the majority of "closed" chaotic quantum systems of quite different microscopic nature shows a great degree of universality on the level of its statistical characteristics of spectra and eigenfunctions stemming from the very basic fact of chaotic internal motion. Because of this universality one achieves the correct description of the properties of such systems by exploiting the similarity with ensembles of large random matrices (RM). The latter principle is a commonly accepted one in the domain of Quantum Chaos 10], see also [11], and recently some sound arguments were given in favour of its general validity 12.

Provided the statistics of the system Hamiltonian is specified, one can work out the S-matrix by standard methods in the theory of quantum scattering [13 and study its statistical characteristics. This way was pioneered by Verbaarschot et al. [5] who calculated the correlation function of S-matrix elements. Other characteristics of the S-matrix and related quantities can be efficiently studied in the framework of this approach as well [14 16,22].

One can also try to make use of the expected universality directly on the level of S-matrix without any reference to the system Hamiltonian. Such a method was developed in a great detail in a series of papers by Mello and collaborators [6]. The probability density for the whole S-matrix can be obtained if one makes the assumption of minimal information content of such a distribution respecting the requirements of S-matrix unitarity and symmetry constraints. Provided all the relevant information about the system is encoded in a value of the average $\mathrm{S}$ matrix $\langle S\rangle$ the probability $P(S)$ was shown to be given by a so-called Poisson's kernel [6]. More recently, Brouwer [17 has shown that the general Poisson kernel can be derived directly from the Hamiltonian approach if one takes the Hamiltonian matrix from a quite specific Lorentzian $\mathrm{RM}$ ensemble. In view of the asymptotic equivalence of the Lorentzian and Gaussian matrices 17] it is natural to expect that the Poisson's kernel is of the same universality as the spectral statistics for closed systems discussed above.

If one wishes to study the dependence of S-matrix on external parameters without explicitly considering the system Hamiltonian, one should make some additional statistical assumptions beyond the minimum information approach. One possible way is to simulate such a dependence by a kind of "Brownian motion" in the corresponding S-matrix space. Quite a detailed presentation of such a method with some attempts to find a microscopic justification of the whole procedure can be found in 18 . It turns out, however, that the Brownian motion picture is in disagreement with the results obtained starting from the Hamiltonian formalism. Therefore, the latter approach seems to be the only consistent one when we are interested in the parametric variations.

Apart from the S-matrix elements, the scattering phase shifts $\theta_{a}$ (defined via the S-matrix eigenvalues $\exp i \theta_{a}$ ) are used intensively to characterize the chaotic scattering, see e.g [3]. Quite recently their statistical characteris- 
tics were studied numerically in some detail 19,20$]$. The derivatives of phase shifts over the energy $\tau_{a}=\partial \theta_{a} / \partial E$ ( which we suggest to call the "phaseshift times") are particularly interesting and related to the mean time spent by a particle in the interaction domain (WignerSmith delay time,see [22] and references therein) as $\tau_{w}=$ $\frac{1}{M} \sum \tau_{a}$. The fluctuations of the individual phaseshift times $\tau_{a}$ can be therefore used to characterize the possible fluctuations of delay times in the scattering process. Let us mention that various aspects of the general problem of delay time characteristics is under quite intensive study for a while [15,21,22]. More general parametric derivatives of the scattering phase shifts can also be related to some observable quantities. As a particular example we mention the relation between the persistent currents and the derivative of the total phase shift over the magnetic flux [23.

In order to be able to study statistics of phase shifts $\theta_{a}$ within the Hamiltonian approach, it is convenient to use the following representation for the scattering matrix (see e.g. [17):

$$
\hat{S}=\left[\hat{I}-i \pi W^{\dagger} \hat{R}(E) W\right] \times\left[\hat{I}+i \pi W^{\dagger} \hat{R}(E) W\right]^{-1}
$$

where we introduced the resolvent $\hat{R}(E)=(E-\mathcal{H})^{-1}$ corresponding to the Hamiltonian $\mathcal{H}$ of the "closed" chaotic system and $W$ is the matrix that couples the chaotic region with the incoming/outgoing waves. In the stochastic approach the system Hamiltonian $\mathcal{H}$ is replaced by the $N \times N$ matrix $H_{i j}$ taken from the corresponding Gaussian RM ensemble. In order to allow for the dependence of the S-matrix on the external parameter $X$ to be taken into account it is convenient to consider the set of RM $H_{i j}$ to be of the form [1] : $\hat{H}(X)=\hat{H}_{0}+\frac{X}{\sqrt{N}} \hat{H}_{1}$. To be specific, we consider in the present paper the simplest possible case when $\hat{H}_{0}$ runs over the Gaussian Unitary Ensemble (GUE) and $\hat{H}_{1}$ is an arbitrary but fixed matrix from the same ensemble. Physically this case is known to describe systems with completely broken time reversal symmetry (TRS). The scattering in systems with partly or completely broken TRS is under quite intensive theoretical [7, 18, 19,24] and experimental [25] study.

The matrix $W$ is $N \times M$ matrix of amplitudes $W_{i a}, a=$ $1,2, \ldots, M$, coupling the internal motion to $M$ open channels. Without much loss of generality these amplitudes can be chosen fixed in a way ensuring that the average $S$-matrix is diagonal in the channel basis: $\overline{S_{a b}}=\delta_{a b} \overline{S_{a a}}$ [5]. Provided the energy $E$ is real, one finds the following expression [5]:

$$
\overline{S_{a a}}=\frac{1-\gamma g(E)}{1+\gamma g(E)} ; \quad \gamma=\pi \sum_{i} W_{i a}^{*} W_{i a}
$$

where $g(E)=i E / 2+\left(1-E^{2} / 4\right)^{1 / 2}$ and we assumed that all $M$ channels are statistically equivalent for the sake of simplicity. The strength of coupling to continua is convenient to be characterized via the "sticking probabilities" (also called the "transmission coefficients" [.
$T_{a}=1-\left|\overline{S_{a a}}\right|^{2}$ which are given for the present case by the following expression:

$$
T_{a}^{-1}=\frac{1}{2}\left[1+\frac{1}{2 \operatorname{Re} g(E)}\left(\gamma+\gamma^{-1}\right)\right]
$$

The quantity $T_{a}$ measures the part of the flux in channel $a$ that spends substantial part of the time in the interaction region [4. [5]. We see that both limits $\gamma \rightarrow 0$ and $\gamma \rightarrow \infty$ equally correspond to the weak effective coupling regime $T_{a} \ll 1$ whereas the strongest coupling (at fixed energy $E$ ) corresponds to the value $\gamma=1$. The maximal possible coupling corresponding to the upper bound $T_{a}=1$ is achieved in the present model for an energy interval in the vicinity of the center $E=0$.

The eq.(1) shows that eigenphases $\theta_{a}$ considered modulo $2 \pi[26]$ are determined in a unique way by the eigenvalues $z_{a}(E, X)$ of the matrix $\hat{A}_{X}(E)=\pi W^{\dagger} \hat{R}_{X}(E) W$ in view of the relation: $\theta_{a}=-2 \arctan z_{a}$. To this end let us introduce the density $\rho_{E, X}(z)=\frac{1}{M} \sum_{a=1}^{M} \delta\left(z-z_{a}(E, X)\right)$ and consider the correlation function:

$$
\begin{gathered}
\mathcal{K}_{E, \Omega, X}\left(z_{1}, z_{2}\right)= \\
\left\langle\rho_{E, 0}\left(z_{1}\right) \rho_{E+\Omega, X}\left(z_{2}\right)\right\rangle-\left\langle\rho_{E, 0}\left(z_{1}\right)\right\rangle\left\langle\rho_{E+\Omega, X}\left(z_{2}\right)\right\rangle
\end{gathered}
$$

where the angular brackets stand for the averaging over the RM ensemble. This correlation function can be easily found provided the following one is known for $\epsilon \rightarrow 0^{+}$:

$$
f\left(z_{1}, z_{2}\right)=\left\langle\operatorname{Tr} \frac{1}{z_{1}-i \epsilon-\hat{A}_{0}(E)} \operatorname{Tr} \frac{1}{z_{2}+i \epsilon-\hat{A}_{X}(E+\Omega)}\right\rangle
$$

A somewhat unpleasant feature of eq.(5) is that the random matrix $\hat{H}$ enters it only via the matrix $\hat{A}$. However, due to the identity

$$
\begin{array}{r}
\operatorname{det}\left(z-\hat{A}_{X}(E)\right)= \\
z^{M} \operatorname{det}\left[\hat{R}_{X}(E)\left(E-\hat{H}(X)-\frac{\pi}{z} W W^{\dagger}\right)\right]
\end{array}
$$

the right hand side of eq.(5) can be written in the following form:

$$
\begin{aligned}
f\left(z_{1}, z_{2}\right) & =\left.\frac{\partial^{2}}{\partial J_{1} \partial J_{2}}\left[\left(\frac{Z_{J}^{(1)} Z_{J}^{(2)}}{Z_{J=0}^{(1)} Z_{J=0}^{(2)}}\right)^{M} \mathcal{F}\left(J_{1}, J_{2}\right)\right]\right|_{J_{1}=J_{2}=0} \\
\mathcal{F}\left(J_{1}, J_{2}\right) & =\left\langle\frac{\operatorname{det}\left[E-H_{e f f}\left(0 ; Z_{J}^{(1)}\right)\right] \operatorname{det}\left[E+\Omega-H_{e f f}\left(X ; Z_{J}^{(2)}\right)\right]}{\operatorname{det}\left[E-H_{\text {eff }}\left(0 ; Z_{J=0}^{(1)}\right)\right] \operatorname{det}\left[E+\Omega-H_{\text {eff }}\left(X ; Z_{J=0}^{(2)}\right)\right]}\right\rangle
\end{aligned}
$$

where we introduced the notations: $Z_{J}^{(p)}=z_{p}-i(-1)^{p} \epsilon+$ $J_{p} ; \quad p=1,2$ and $H_{e f f}\left(X ; Z_{J}^{(p)}\right)=\hat{H}(X)+\frac{\pi}{Z_{J}^{(p)}} W W^{\dagger}$. In the latter form the expression is very close in its structure to that used to study parametric correlations in ensembles of large RM 111 and can be evaluated by essentially the same method. The general strategy is as follows: 1) to represent determinants in the denominator 
of the preceeding expression by auxilliary Gaussian integrals over commuting variables and those in the numerator by similar integrals over anticommuting variables. 2) To perform the averaging over the GUE distribution of $\left.H_{0} .3\right)$ After employing the Hubbard-Stratonovich transformation to exploit the limit $N \gg M$ when integrating out some ("massive") degrees of freedom in the saddlepoint approximation. After this is done the integral over the remaining ("massless") degrees of freedom can be represented in a form of the so-called zero-dimensional supermatrix nonlinear $\sigma$-model introduced for the first time by Efetov [27].

For the connected part of the correlation function eq.(4), we find after the set of standard manipulations [5,11] the following expression:

$$
\begin{gathered}
f\left(z_{1}, z_{2}\right)=\int_{-1}^{1} d \lambda \int_{1}^{\infty} d \lambda_{1} \frac{\mathcal{F}_{M}\left(\lambda, \lambda_{1}\right)}{\left(\lambda_{1}-\lambda\right)^{2}} \times \\
\exp \left\{i \omega\left(\lambda_{1}-\lambda\right)-\frac{x^{2}}{2}\left(\lambda_{1}^{2}-\lambda^{2}\right)\right\} \\
\mathcal{F}_{M}\left(\lambda_{1}, \lambda\right)=\frac{\partial^{2}}{\partial z_{1} \partial z_{2}}\left[\frac{z_{1} z_{2}+\gamma^{2}+i \gamma\left(z_{1}-z_{2}\right) \lambda}{z_{1} z_{2}+\gamma^{2}+i \gamma\left(z_{1}-z_{2}\right) \lambda_{1}}\right]^{M}
\end{gathered}
$$

where we put for simplicity the energy parameter $E=0$ and made the natural rescaling [1]]: $\omega=\pi \Omega / \Delta ; \quad x^{2}=$ $(\pi X / N \Delta)^{2} \frac{1}{N} \operatorname{Tr} \hat{H}_{1}^{2}$, with $\Delta \propto 1 / N$ being the mean level spacing of the GUE at $E=0$.

The eq.(7) describes the parametric correlations of eigenvalues of the matrix $\hat{A}_{X}(E)$ in closed form and provides the basis for extracting the statistical properties of scattering phase shifts. First of all, it is evident that the integrations in eq.(7) can be performed explicitly when $x=\omega=0$ thus giving the expression for the correlation function of phase shifts at fixed value of the energy $E$ and the parameter $X$. In order to represent it in a simple form for any value of the coupling constant $\gamma$ it is convenient to introduce new variables $\phi$ related to the scattering phase shifts $\theta$ as $\phi=\arctan \left\{\gamma^{-1} \tan (\theta / 2)\right\}$. A direct calculation shows that the correlation function of the densities of the angles $\phi$ is equal to

$$
\left.\mathcal{K}\left(\phi_{1}, \phi_{2}\right)\right|_{\phi_{1} \neq \phi_{2}}=-\left(\frac{\sin M\left(\phi_{1}-\phi_{2}\right)}{\pi M \sin \left(\phi_{1}-\phi_{2}\right)}\right)^{2}
$$

for any number of open channels. Remembering the relation between $\gamma$ and $\langle S\rangle$, see eq.(2), one can satisfy oneself that eq.(8) exactly coincides with the pair correlation function following from the Poisson's kernel distribution as defined in 66,17.

Let us turn our attention to the distributions of the individual phaseshift times $\tau_{a}=\partial \theta_{a} / \partial E$ and general parametric derivatives of phase shifts. In view of the relation $\theta_{a}=-2 \arctan z_{a}$ these distributions can be found easily provided the joint probability densities $\mathcal{P}_{E}(z, t)$ and $\mathcal{P}_{X}(z, y)$ of $z_{a}$ and one of its parametric derivatives - $t_{a}=\partial z_{a} / \partial E$ or $y_{a}=\partial z_{a} / \partial X-$ is known. The functions $\mathcal{P}_{E}$ and $\mathcal{P}_{X}$ can be determined from the correlation function eq.(4) as follows (see e.g. [28):

$$
\begin{array}{r}
\mathcal{P}_{E}(z, t)=\frac{1}{M}\left\langle\sum_{a=1}^{M} \delta\left(z-z_{a}\right) \delta\left(t-\partial z_{a} / \partial E\right)\right\rangle \\
=M \lim _{\Omega \rightarrow 0} \Omega \mathcal{K}_{E, \Omega, X=0}\left(z_{1}=z, z_{2}=z+t \Omega\right) \\
\mathcal{P}_{X}(z, y)=\frac{1}{M}\left\langle\sum_{a=1}^{M} \delta\left(z-z_{a}\right) \delta\left(y-\partial z_{a} / \partial X\right)\right\rangle \\
=M \lim _{X \rightarrow 0} X \mathcal{K}_{E, \Omega=0, X}\left(z_{1}=z, z_{2}=z+y X\right)
\end{array}
$$

Using the procedure described above we find the following distribution of inverse (scaled) phaseshift times $u=2 \pi /(\tau \Delta)$ :

$$
\begin{array}{r}
\mathcal{P}_{u}(u) \equiv \frac{1}{M}\left\langle\sum_{a=1}^{M} \delta\left[u-2 \pi /\left(\tau_{a} \Delta\right)\right]\right\rangle \\
=\frac{(-1)^{M}}{M !} u^{M} \frac{d^{M}}{d u^{M}}\left(e^{-g u} I_{0}\left(u \sqrt{g^{2}-1}\right)\right)
\end{array}
$$

where $I_{0}(z)$ stands for the modified Bessel function, $g=2 T^{-1}-1$ and $T$ is the "sticking probability" defined earlier. Similarly, the scaled parametric derivatives $v_{a}=\partial \theta_{a} / \partial x$ are found to be distributed according to the probability density

$$
\mathcal{P}_{v}(v)=\int_{0}^{\infty} \frac{d u u}{(2 \pi)^{1 / 2}} \exp \left[-\frac{u^{2} v^{2}}{2}\right] \mathcal{P}_{u}(u)
$$

where $\mathcal{P}_{u}(u)$ is defined in the preceeding equation. Both distributions depend on the coupling constant $\gamma$ only via the sticking probability $T$ rather than via the average $\mathrm{S}$ matrix. They assume the simplest form for the "critical" coupling $T=1$ corresponding to the most strong overlap of individual resonances allowed for the few-channel scattering 15,16]. Under this condition one finds the following distribution of scaled phaseshift times $\tau_{s}=1 / u$ : $\mathcal{P}_{\tau}\left(\tau_{s}\right)=a=\frac{1}{M !} \tau_{s}^{-M-2} e^{-1 / \tau_{s}}$.

In the recent paper 20] this formula was found to be in a good agreement with results of direct numerical simulations. The authors also found a way to arrive at such an expression exploiting some plausible assumptions about the statistics of S-matrix eigenphases and their derivatives.

Having at our disposal the exact distribution eq.(11) it is instructive to calculate the mean value and the variance of the phaseshift times. One finds:

$$
\langle\tau\rangle=\frac{2 \pi}{M \Delta} ; \quad \frac{\left\langle\tau^{2}\right\rangle-\langle\tau\rangle^{2}}{\langle\tau\rangle^{2}}=\frac{2 M\left(T^{-1}-1\right)+1}{M-1}
$$

The first of these relations is quite well known [21,22]. It shows that the mean delay time $\langle\tau\rangle$ is determined by the mean level spacing $\Delta$ of the closed system and the number $M$ of open channels. On the other hand the magnitude of delay time fluctuations measured by the relative variance of the phaseshift time distribution, see eq. (13), is determined both by $M$ and $T$. Generically, 
the fluctuations are the weaker the larger is the number of open channels $M$ and the stronger is the coupling to continua: $1-T \ll T$. Let us also mention as an interesting feature the divergency of the time-delay variance at $M=1$, which is a consequence of the powerlaw tail $\tau_{s}^{-M-2}$ typical for the distribution $\mathcal{P}_{\tau}\left(\tau_{s}\right)$.

Eq.(7) can be used to study the parametric correlation functions of the Wigner-Smith delay times $\tau_{w}(E, X)$. However, this calculation turns out to be quite lengthy and will be presented in a more extended publication. On the other hand, remembering the definition $\tau_{W}=-\frac{i}{M} \frac{\partial}{\partial E} \ln \operatorname{det} S(E)$ one finds the relation $\tau_{w}=$ $-\frac{2}{M} \operatorname{Im} \operatorname{Tr}\left(E-\mathcal{H}+i \pi W W^{\dagger}\right)^{-1}$ which follows directly from eq.(1) upon using the identity eq.(6). Now it is evident that the calculation of the correlation of fluctuations of Wigner-Smith delay times $\delta \tau_{W}(E, X)=\tau_{W}-$ $\left\langle\tau_{W}\right\rangle$ amounts to evaluating the average product of the resolvents of the non-Hermitian effective Hamiltonians $\mathcal{H} \pm i \pi W W^{+}$. For the case of chaotic systems with preserved TRS such a calculation was done earlier in [22]. For the present case we find:

$$
\begin{array}{r}
\left\langle\delta \tau_{W}(E, 0) \delta \tau_{W}(E+\Omega, X)\right\rangle= \\
2\left(\frac{\pi}{M \Delta}\right)^{2} \times \int_{-1}^{1} d \lambda \int_{1}^{\infty} d \lambda_{1} \cos \omega\left(\lambda_{1}-\lambda\right) \\
\exp \left\{-\frac{x^{2}}{2}\left(\lambda_{1}^{2}-\lambda^{2}\right)\right\} \times\left[\frac{1+\lambda g^{-1}}{1+\lambda_{1} g^{-1}}\right]^{M}
\end{array}
$$

The variance of the $\tau_{w}$ is given by:

$$
\frac{\left\langle\tau_{W}^{2}\right\rangle-\left\langle\tau_{W}\right\rangle^{2}}{\left\langle\tau_{W}\right\rangle^{2}}=\frac{2}{T^{2}\left(M^{2}-1\right)}\left[1-(1-T)^{M+1}\right]
$$

showing the same qualitative features (divergencies at $M=1$ or $T \rightarrow 0)$ as those following from the eq.(13).

In conclusion, we studied analytically the parametric correlations of scattering phase shifts and the distribution of phase shift derivatives for a chaotic quantum system with broken TRS. The method of calculation automatically provides the universality of the obtained results for a very broad class of systems which can be described by the mapping on the same supermatrix nonlinear $\sigma-$ model [11. Another important feature of the method is that it provides the regular basis for studying a general case of disordered systems where the effects of localization modify essentially the phaseshift statistics [19]. This issue as well as the extension of our results to the case of systems with preserved TRS are left for future research.

Authors are very obliged to P.Seba,J.Zakrzewski and K.Zyczkowski for providing them with the text of the paper 20 prior to publication. Y.V.F. appreciates stimulating and clarifying discussions on chaotic scattering with P.Seba and is grateful to N.Lehmann for useful discussions. The financial support by SFB 237 "Unordnung und grosse Fluktuationen" is acknowledged with thanks.
* on leave from: Petersburg Nuclear Physics Institute, Gatchina 188350, St.Petersburg District, Russia

[1] U.Smilansky in Chaos and Quantum Physics. Proceedings of the Les-Houches Summer School. Session LII, ed. by M.J.Giannoni et.al (North Holland, Amsterdam, 1991),p.372

[2] P.Gaspard in: Quantum Chaos. Proceedings of E. Fermi Summer School, ed.by G.Casati et.al. (1991), p.307

[3] R.Blümel, U.Smilansky Phys.Rev.Lett. v.64 (1989) 241; E.Doron and U.Smilansky Nonlinearity v.5 (1992) 1055

[4] C.H.Lewenkopf and H.A.Weidenmüller, Ann.Phys. v.212 (1991),53

[5] J.J.M.Verbaarschot,H.A.Weidenmüller and M.R.Zirnbauer, Phys.Rep v.129 (1985), 367;

[6] P.Mello, P.Pereyra and T.H.Seligman, Ann.Phys. (NY) v.161 (1985), 254; P.Mello, H.Baranger Physica A v.220 (1995), 15 and references therein.

[7] R.A.Jalabert, A.D.Stone and Y.Alhassid Phys.Rev.Lett. v.68 (1992), 3468

[8] C.M.Marcus et al. Phys.Rev.Lett.v.69 (1992) 506

[9] H.J.Stöckmann and J.Stein, Phys.Rev.Lett. v.64, (1990) 2215; S.Sridhar et.al J.Stat.Phys. v.68 (1992), 239; H.Alt et al Phys.Rev.Lett. v.74 (1995),62.

[10] O.Bohigas, in Chaos and Quantum Physics, see Ref. [1]

[11] B.L.Altshuler and B.D.Simon in: Mesoscopic Quantum Physics ed. by E.Akkermans et al, Les Houches Summer School,Session LXI

[12] B.A. Muzykantsky, D.E.Khmelnitsky JETP Lett v.62 (1995) 76; A.V.Andreev et al. cond-mat/960100

[13] C.Mahaux and H.A.Weidenmüller, Shell Model Approach in Nuclear Reactions (North-Holland, Amsterdam,1969)

[14] A.M.S. Macedo Phys.Rev. E v.50 (1994) R659; H.Bruus et al cond-mat/951011.

[15] H.L.Harney et.al Ann.Phys. v.220 (1992),159; F.M.Izrailev et. al Phys.Rev.E v.49 (1994),130

[16] Y.V. Fyodorov, H.-J. Sommers cond-mat/9507117.

[17] P.W.Brouwer Phys.Rev.B v.51 (1995) 16875

[18] K.Frahm, J.-L.Pichard J. Phys.I France v.5 (1995) 847; J.Rau Phys.Rev.B v.51(1995), 7734

[19] R.A.Jalabert, J.-L.Pichard J. Phys.I France v.5 (1995) 287

[20] P.Seba , K.Zyczkowski, J.Zakrzewski , chao-dyn 9603005

[21] V.L.Luboshitz Yad.Fiz. v.37 (1983) 292; G.Ionnacone Phys.Rev.B v.51 (1995) 4727;

[22] N.Lehmann et al. Physica D v.86 (1995) 572

[23] E.Akkermans et al. Phys.Rev.Lett. v.66 (1991) 76

[24] Y.Wang et al. Phys. Rev.B v.49 (1994) 1928; Z.Pluhar et al.Ann.Phys.(NY) v.243 (1995),1; X.Yang et al Phys.Rev.B v. 52 (1995), 8219; Z.Yan ,R.Harris Europh.Lett. v.32 (1995) p.437; O.Bohigas et al. Nonlinearity v.8 (1995), 203

[25] U.Sivan et.al Europh.Lett v.25 (1994) 605;P.So et.al Phys.Rev.Lett. v.74 (1995), 2662; U.Stoffregen et.al ibid. 2667; J.A.Folk et.al (Phys.Rev.Lett) v.76 (1996),1699

[26] Actually $\theta_{a}(E)=2\left(\pi N \int_{-\infty}^{E} \rho(E) d E-\arctan z_{a}\right)$, where $\rho(E)$ is the density of states for closed system.

[27] K.B.Efetov, Adv.Phys. v.32 (1983),53;

[28] V.E.Kravtsov, M.R.Zirnbauer, Phys.Rev.B v.46 (1992), 4332 